\title{
Planejamento de atracação de navios por meio de um modelo matemático para o problema de alocação de berços contínuos com descontinuidade do cais
}

\author{
Pedro Hastenreiter Leite Rodrigues ${ }^{1}$, Rodrigo de Alvarenga Rosa르, Bianca Passos Arpini \\ Alexandre Luís Cardoso Bissoli ${ }^{4}$ e Glaydston Mattos Ribeiro ${ }^{4}$
}

\begin{abstract}
Resumo: Os portos brasileiros movimentam anualmente 927 milhões de toneladas de carga que representam 94\% das exportações. Este artigo apresenta um modelo matemático que tem como objetivo contribuir com o planejamento da atracação de navios nos portos, sobretudo, os que têm a característica de ter descontinuidades ao longo do cais causadas ou pela expansão de um trecho que ficou mais largo do que o restante ou pelo porto ter duas margens. O modelo proposto foi denominado de Problema de Alocação de Berços Contínuos com Descontinuidade de Cais (PABC-DC) e foi aplicado nos terminais públicos do Porto de Vitória (Cais Comercial de Vitória e Terminal CODESA). O modelo foi resolvido no CPLEX 12.6 com dados reais do Porto de Vitória. Os resultados do modelo, quando comparados com os resultados do planejamento manual realizado pelo porto, mostraram sempre uma redução no tempo de estadia dos navios no porto chegando a 8,7\%.
\end{abstract}

Palavras-chave: Problema de Alocação de Berços Contínuos com Descontinuidade, Operação Portuária. Programação Linear Inteira Mista.

Abstract: Brazilian ports handle 927 million tons of cargo/year representing 94\% of exports. This paper presents a mathematical model that aims to contribute to the planning of berthing of ships in ports, especially those that have the characteristic of having discontinuities along the wharf or caused by expansion of a passage that was wider than the rest or the port has two margins. The proposed model was called the Continuous Berth Allocation Problem with Discontinuity of Pier (PABC-DC) and was applied in the public terminals at the Port of Vitória (Victory Commercial Wharf and Terminal CODESA). The model was solved in CPLEX 12.6 with real data of Port of Vitória. The model results compared with the results of manual planning done by the port always showed always a reduction in the total time that the ships spent at the port, reaching up $8.7 \%$ reduction.

Keywords: Continuous Berth Allocation Problem with Berth Discontinuities, Port Operation. Mixed Integer Linear Programming.

\section{INTRODUÇÃo}

Os portos brasileiros movimentam anualmente cerca de 927 milhões de toneladas das mais diversas mercadorias que correspondem a mais de $94 \%$ das exportações brasileiras (ANTAQ, 2013).

O Porto de Vitória é considerado um dos mais importantes do Brasil, pela diversidade e volume de cargas transportadas e é administrado pela Companhia Docas do Espírito Santo - CODESA. Ele possui uma boa infraestrutura de transportes ferroviário, rodoviário e marítimo, exercendo substancial influência na economia do Estado do Espírito Santo e do país. A Figura 1a mostra uma vista aérea do Porto de Vitória; na parte superior tem-se o Cais de Vitória e na parte inferior, tem-se o Terminal CODESA. Os dois terminais movimentam diversos tipos de carga, dentre elas, destacam-se: bobinas de papel, celulose, açúcar, produtos agrícolas e siderúrgicos.

\footnotetext{
${ }^{1}$ Pedro Hastenreiter Leite Rodrigues, Departamento de Engenharia de Produção, UFES. (phastenreiter@gmail.com)

2 Rodrigo de Alvarenga Rosa, Programa de Pós-Graduação em

Engenharia Civil, UFES. (rodrigo.a.rosa@ufes.br)

3 Bianca Passos Arpini, Programa de Pós-Graduação em Engenharia

Civil, UFES. (bianca.p.arpini@gmail.com)

${ }^{4}$ Alexandre Luís Cardoso Bissoli, Departamento de Engenharia Civil,

UFES. (xandybissoli.5@hotmail.com)

${ }^{4}$ Glaydston Mattos Ribeiro, Programa de Pós-Graduação em Engenharia de Transportes, UFRJ. (glaydston@pet.coppe.ufrj.br)
}

Manuscrito recebido em 26/10/2014 e aprovado para publicação em 19/12/2014.

Este artigo é parte de TRANSPORTES v. 23, n. 1, 2015. ISSN: 2237-1346

(online). DOI:10.4237/transportes.v23i1.861.
O Cais Comercial de Vitória possui quatro berços de atracação denominados 101, 102, 103 e 104 localizados na margem correspondente à cidade de Vitória. Entretanto, o berço 104 apresenta poucas atracações devido à sua reduzida profundidade. Assim, as análises apresentadas neste artigo desconsideraram este berço. Com isso, o Cais Comercial de Vitória considerado corresponde aos berços 101, 102 e 103, que tem 587,0 m de extensão. Na Figura 1b destacase a descontinuidade do Cais Comercial entre os berços 101 e 102 e o berço 103 que foi gerada em função da expansão da largura do cais ao longo do berço 101 e 102. A descontinuidade do Cais Comercial de Vitória é tratada nesse artigo como Descontinuidade 1.

O Terminal CODESA possui dois berços de atracação, 201 e 202, com 427,0 m de extensão e estão localizados na margem do lado da cidade de Vila Velha, oposta ao cais Comercial de Vitória.

Apesar de haver uma denominação de diferentes berços nos dois terminais, a CODESA opera ambos como se fossem contínuos, ou seja, ela atraca o navio no cais independente do tamanho de cada berço, distribuindo os navios ao longo de toda extensão do cais. Como os navios que chegam ao Porto de Vitória podem atracar nas duas margens do porto, deve-se considerar os dois terminais como opções de atracação e, então, surge uma nova descontinuidade no problema que é a atracação em margens diferentes. Para fins de modelagem matemática do processo de atendimento dos navios, os terminais podem ser considerados como se estivessem na mesma margem, desta forma, considera-se que o final do Cais Comercial de Vitória estaria continuando no Terminal CODESA. Esta consideração de conexão dos dois terminais em duas margens separadas é, neste artigo, denominada Descontinuidade 2. 


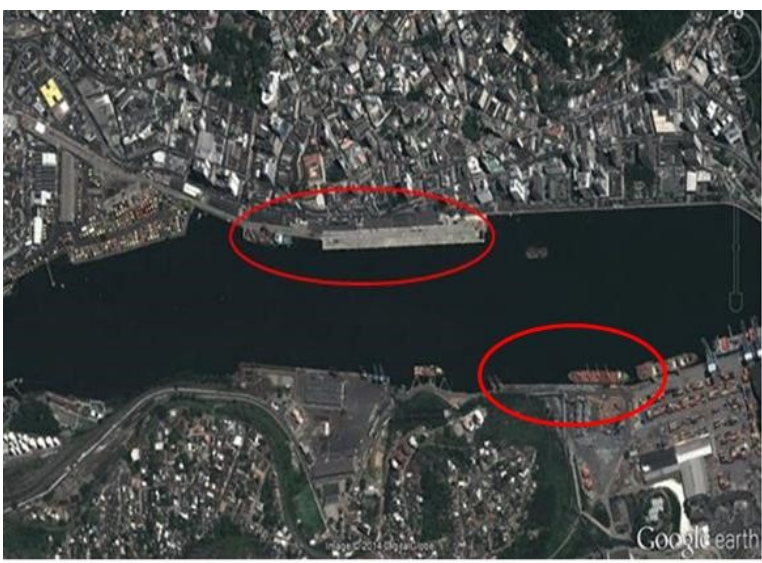

a

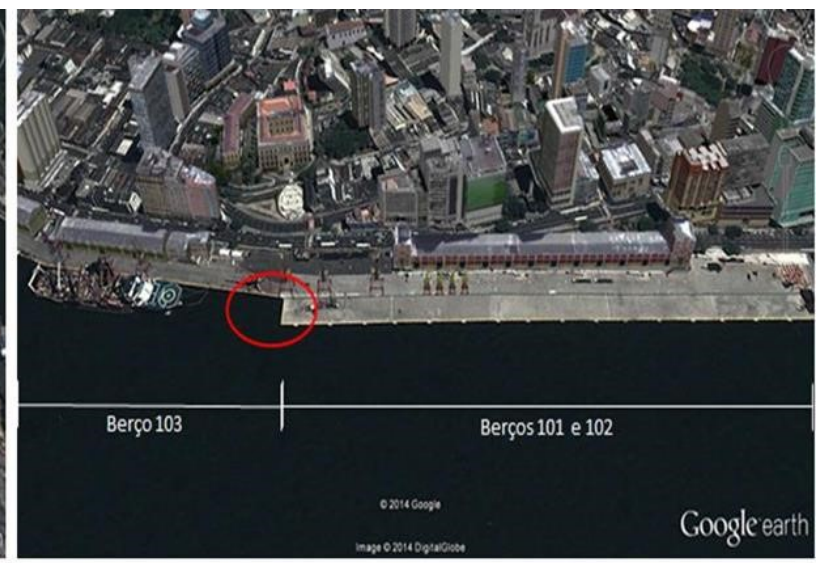

b

Figura 1. a) Vista aérea porto - Cais Comercial de Vitória parte de cima e Terminal CODESA parte de baixo; b) Detalhe da Descontinuidade do Cais Comercial

A partir dessa consideração, utiliza-se a expressão Cais Total quando para se referir à soma da extensão do Cais Comercial de Vitória com a extensão do Terminal CODESA. O Cais Total possui 1014,0 m de extensão. Na Figura 2a pode-se ver um layout do Cais Total com as duas descontinuidades citadas.

Assim, o planejamento de atendimento dos navios que chegam ao Porto de Vitória para atracar no Cais Comercial ou no Terminal CODESA deve respeitar as descontinuidades do Cais Total. Os navios devem ser atracados de tal forma que seu comprimento total fique entre as descontinuidades. A Figura 2b apresenta duas atracações que não podem ocorrer, pois existem dois navios que estão atracados sobre a descontinuidade do cais. Já a Figura 2c apresenta alguns exemplos de atracações que respeitam as descontinuidades do cais. Por outro lado, os navios possuem restrição temporal, isto é, cada navio possui uma data de chegada e não pode ser atracado antes dessa data.
Este artigo propõe um modelo matemático, denominado de Problema de Alocação de Berços Contínuo com Descontinuidade de Cais (PABC-DC), para o planejamento de atracação de navios em um porto público que visa resolver o problema descrito anteriormente com o objetivo de reduzir o tempo total que os navios ficam a disposição do porto, ou seja, reduzir a diferença entre os momentos de desatracação e os instantes de chegada. Os resultados alcançados pelo modelo, quando comparados com aqueles obtidos pelo planejamento manual realizado pelo porto, mostraram sempre uma redução no tempo de estadia dos navios no porto chegando a $8,7 \%$.

É importante mencionar que o modelo pode ser aplicado a diversos portos do Brasil e do mundo que possuam duas margens ou qualquer detalhe de projeto que impeça a atracação de forma contínua.

O artigo é organizado como segue. Na Seção 2 temse a definição do Problema de Alocação de Berço Contínuo (PABC) e uma revisão da literatura. A Seção 3 apresenta o

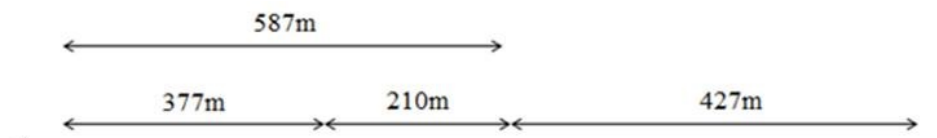

a)
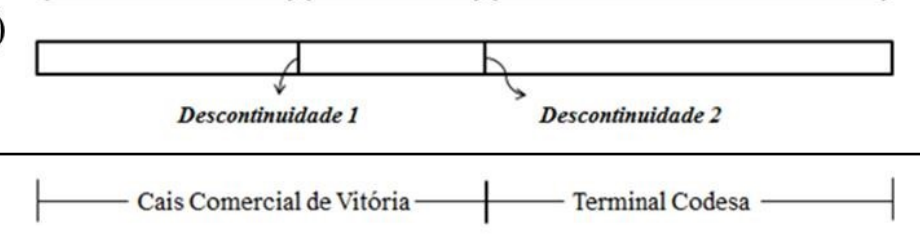

b)
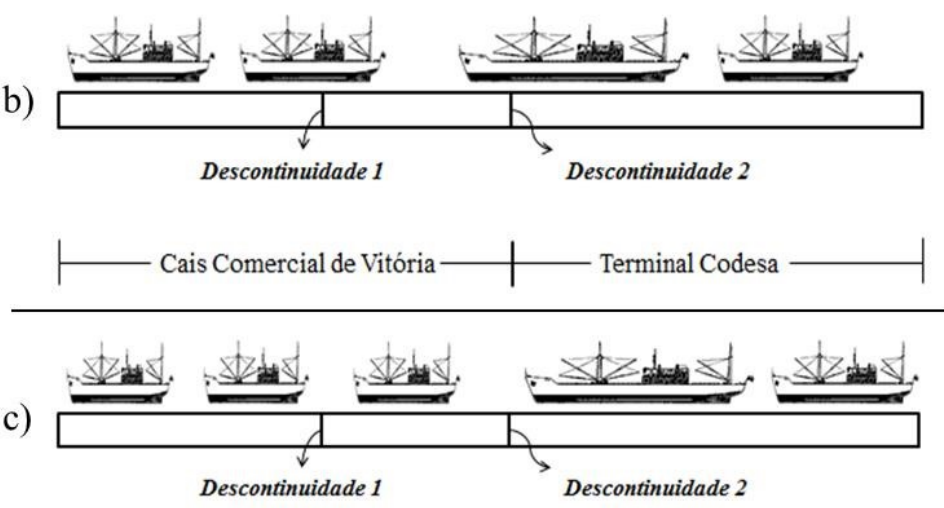

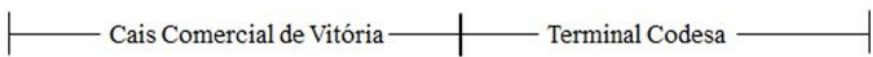

Figura 2. Layout do Cais Total; b) Situações Inviáveis - Descontinuidade 1 e 2 não respeitadas; c) Situação Viável - Descontinuidades respeitadas 
Planejamento de Atracação de Navios por Meio de um Modelo Matemático para o

Problema de Alocação de Berços Contínuos com Descontinuidade do Cais
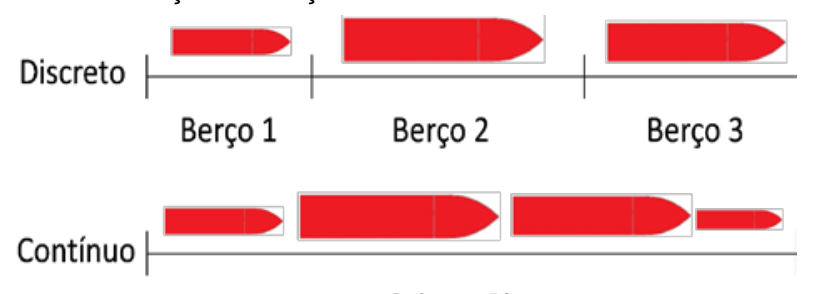

Cais ou Pier

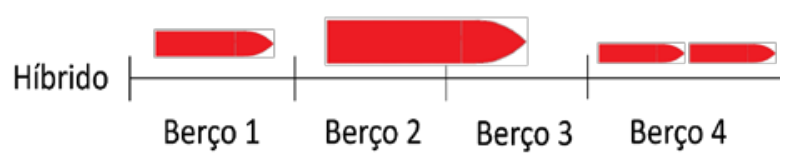

Figura 3. Representação $P A B D, P A B C$ e $P A B H$

modelo matemático proposto. A Seção 4 descreve a metodologia, a apresentação e as análises dos resultados encontrados e, por último, a Seção 5 apresenta as principais conclusões do trabalho.

\section{PROBLemA DE ALOCAÇÃo DE BERÇOS}

O Problema de Alocação de Berços (PAB) refere-se ao problema de alocar os navios que solicitam atracação no porto a uma posição de atracação no cais determinando a posição e o horário de atracação. Ou seja, não se pode autorizar a ocupação de mais de um navio para um mesmo espaço no cais num mesmo instante.

Existem vários objetivos para o $\mathrm{PAB}$ tais como minimização do tempo de estadia no porto, minimização do desvio entre a escala de atracação real e a planejada, e maximização do número de navios atendidos (Bierwirth e Meisel, 2009). Devido à sua importância, o PAB tem recebido considerável atenção da comunidade acadêmica e há diversos artigos com revisões sobre o tema (Meersmans e Dekker, 2001; Vis e de Koster, 2003; Steenken et al., 2004; Vacca et al., 2007; Stahlbock e Voss, 2008; Bierwirth e Meisel, 2010 e Rashidi e Tsang, 2013).

Existem várias restrições envolvidas na alocação de berços, o que pode levar a diferentes formulações e modelos. Conforme a literatura, os modelos existentes para o estudo do PAB trabalham com restrições temporais e restrições espaciais (Bierwirth e Meisel, 2009). As restrições espaciais limitam as possibilidades de variar as posições dos navios no berço de acordo com a divisão pré-definida desses elementos no cais. Segundo Imai et al. (2005), os casos de restrições espaciais consistem em: PAB Discreto (PABD); PAB Contínuo (PABC) e PAB Híbrido (PABH).

Tanto o PABD quanto o PABH possuem o cais dividido em um dado número de seções, denominadas berços. No PABD, o navio ocupa um único berço por vez, já o $\mathrm{PABH}$ permite que um navio de maior porte ocupe mais do que um berço, e ainda, que parte de um berço pode ser ocupada com um navio de menor porte (Bierwirth e Meisel, 2009).

O PABC, por sua vez, não tem o cais dividido em berços. Dessa forma, os navios podem ocupar qualquer posição ao longo do cais, respeitando os seus limites, o que garante maior flexibilidade do espaço de atracação. Ao contrário do que possa parecer, este layout torna mais difícil o planejamento quando comparado ao PABD. Isto porque pode levar ao aparecimento de espaços sem utilização nos cais e não permitir que navios de certas dimensões atraquem
(Bierwirth e Meisel, 2009). A Figura 3 mostra uma representação gráfica de cais de layouts diferentes.

Além disso, na formulação do PAB as restrições de calado dos navios podem ser consideradas limitantes na escolha das possíveis posições de atracação ou berços, pois estes, no caso do PABC, devem ter uma profundidade maior do que o calado do navio. Deve-se ainda assegurar que o comprimento do navio seja menor do que o espaço disponível para atracação.

No que tange às restrições temporais, as datas e horários de atracação e desatracação dos navios parâmetros importantes. Segundo Imai et al. (2001), o processo de chegada pode ser classificado como 1) Estático ou 2) Dinâmico. Na chegada estática não há considerações quanto aos horários de chegada dos navios, pois assume-se que todos os navios estão aguardando na área de fundeio do porto e podem atracar imediatamente. Na chegada dinâmica, considera-se que os navios têm horários definidos de chegada e não têm permissão para atracar antes do horário previsto (Bierwirth e Meisel, 2009).

O gráfico espaço-tempo para o PABC, Figura 4, é utilizado para apresentar graficamente o planejamento das atracações dos navios no porto. O eixo vertical corresponde ao comprimento do cais, enquanto o eixo horizontal representa o horizonte de tempo de planejamento. Cada retângulo representa a atracação de um navio no porto. A altura deste retângulo representa o comprimento do navio e a largura o tempo de operação do navio. As coordenadas inferiores à esquerda de cada retângulo representam a localização de atracação no cais e o instante da atracação. É inviável que duas embarcações se sobreponham no espaço e/ou no tempo. Assim, em uma solução viável do PAB, todos os retângulos (navios) não devem se sobrepor e cada navio deve respeitar as limitações espaciais e temporais ao longo do cais (Umang e Bierlaire, 2012).

Quanto ao PABC, Garey e Johnson (1979) o representaram como um problema de corte bidimensional com algumas restrições adicionais. Elwany et al. (2013) propuseram uma solução baseada em Simulated Annealing que considera chegada dinâmica e restrições de calado. Li et al. (1998), Guan et al. (2002), Park e Kim (2003) e Guan e Cheung (2004) propuseram soluções para o PABC com chegada estática, com objetivo de minimizar o tempo total de permanência dos navios no porto. Lim (1998), Lim (1999), Tong et al. (1999) e Goh e Lim (2000) consideraram chegada estática e que as posições de atracação deviam ser decididas visando minimizar o comprimento de cais necessário para atracar todos os navios. A minimização de atrasos como função objetivo foi tratada por Moon (2000), Park e 


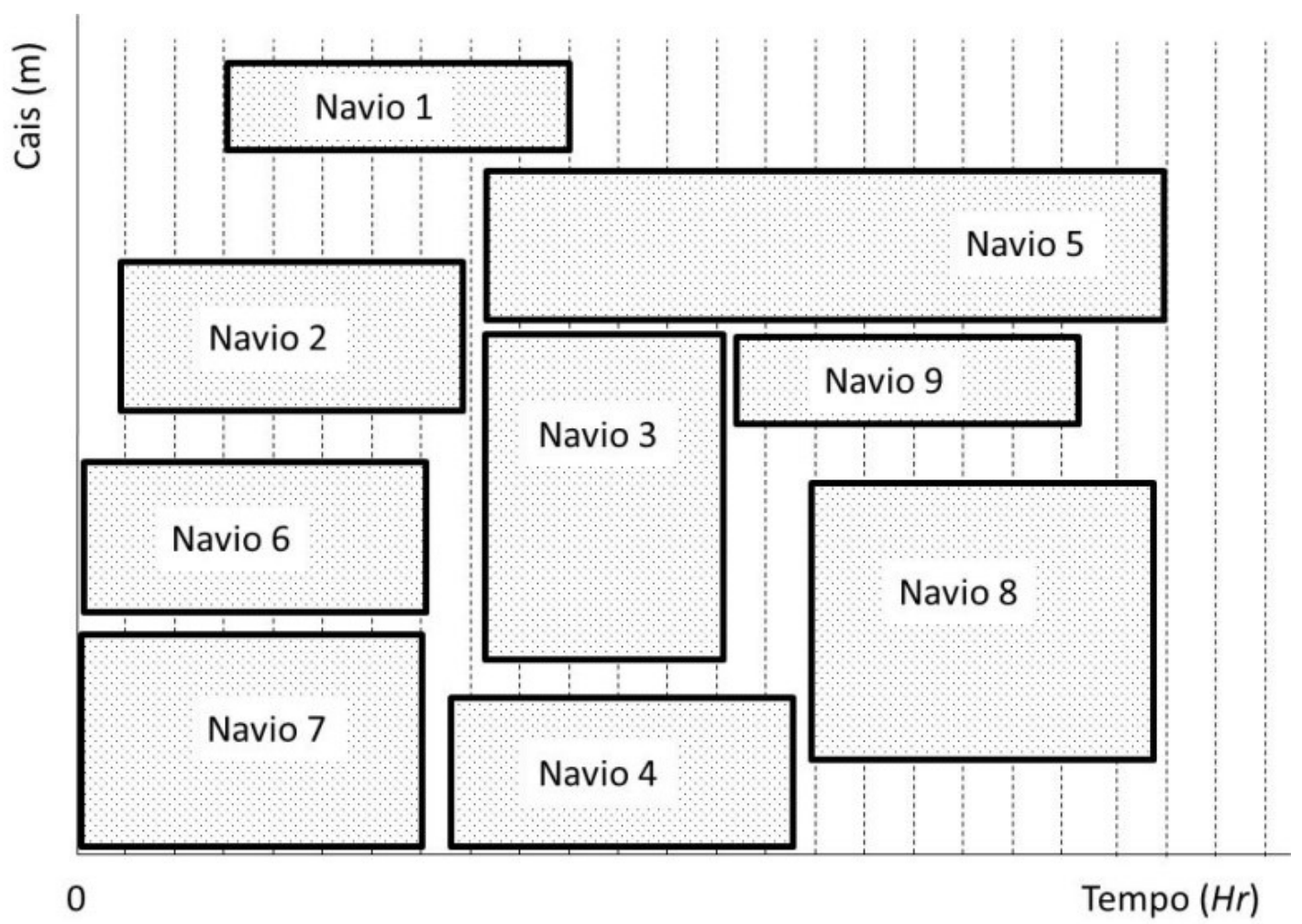

Figura 4. Gráfico espaço-tempo para o PABC

Kim (2002 e 2003), Kim e Moon (2003) e Briano et al. (2005). Estes últimos utilizaram Relaxação Langrangiana e Simulated Annealing.

Imai et al. (2005) e Chang et al. (2008) consideraram que o tempo de carregamento é dependente da posição de atracação do navio no berço contínuo e, também, as restrições de calado. Wang e Lim (2007) buscaram a minimização dos custos de penalização para os navios rejeitados e apresentaram uma heurística robusta que consegue resolver instâncias com até 400 navios. Bierwirth e Meisel (2009, 2010) além do PAB, consideraram os problemas de alocação de guindastes para terminais de contêineres. Brown et al. $(1994,1997)$ e Lee e Chen (2008) propuseram um caso raro na prática no qual os navios podem ser movimentados durante a operação de um berço para o outro. Park e Kim (2002) e Kim e Moon (2003) abordaram o PABC por Relaxação Lagrangiana e testaram instâncias com até 20 navios.

Kim e Park (2004) utilizaram Greedy Randomized Adaptative Search Procedure (GRASP) para resolver o PABC considerando os guindastes do cais. Ganji et al. (2010) propuseram minimizar o tempo de espera por meio de um modelo em que os navios apresentam um ponto ótimo de atracação no cais e aplicaram penalizações devido a disparidade do ponto de atracação e o ponto ótimo.

Em relação ao PABH, Moorthy e Teo (2006) e Chen e Hsieh (1999) estudaram o problema com data de chegada dinâmica, considerando o tempo de carregamento como fixo e a data de chegada dos navios de forma estocástica. Cordeau et al. (2005) e Imai et al. (2007) consideraram que o tempo de operação é dependente da posição de atracação do navio. Nishimura et al. (2001) e Cheong et al. (2010) consideraram restrições de calado e chegada dinâmica. Dai et al. (2008) pesquisaram as posições de atracação dentro das áreas disponíveis usando Simulated Annealing. Chen e Hsieh (1999) propuseram um modelo de Programação Linear Inteira Mista (PLIM) considerando chegadas dinâmi- cas. Hoffarth e Voss (1994), Nishimura et al. (2001), Cordeau et al. (2005), Imai et al. (2007) e Cheong et al. (2007) estudaram o PABH com diversas variações de função objetivo e de restrições. Umang et al. (2013) apresentaram o PABH com data de chegada dinâmica para o caso de portos a graneis.

Problemas semelhantes ao estudado neste artigo foram abordados por Cordeau et al. (2005) e Tang et al. (2009). Cordeau et al. (2005) propuseram um Tabu Search que pode resolver o problema de descontinuidade do cais, especificamente locais que tenham curvas acentuadas. No entanto, eles não apresentaram um modelo matemático para essa situação, nem apresentaram resultados específicos para casos de descontinuidades de cais. Tang et al. (2008) propuseram um modelo PABC para portos que possuem dois segmentos de berços, podendo atracar os navios nesses segmentos. Eles propuseram uma Relaxação Lagrangiana para resolver um caso real de um porto que opera carga a granel. O modelo proposto neste artigo considera o cais como uma única extensão com limitações na descontinuidade, já o modelo de Tang et al.(2008) trata cada parte do cais como berços discretos.

\section{MODELO MATEMÁTICO PROPOSTO}

O modelo matemático proposto para o Caso do Porto de Vitória foi elaborado como um modelo de Programação Linear Inteira Mista (PLIM) e tem como base a teoria de corte e empacotamento bidimensional. Ele tem como objetivo a minimização do tempo total de permanência do navio no porto, calculado pela diferença entre o horário de desatracação e o horário de chegada do navio no porto. As datas de chegada dos navios têm comportamento determinístico. 
Planejamento de Atracação de Navios por Meio de um Modelo Matemático para o

Problema de Alocação de Berços Contínuos com Descontinuidade do Cais

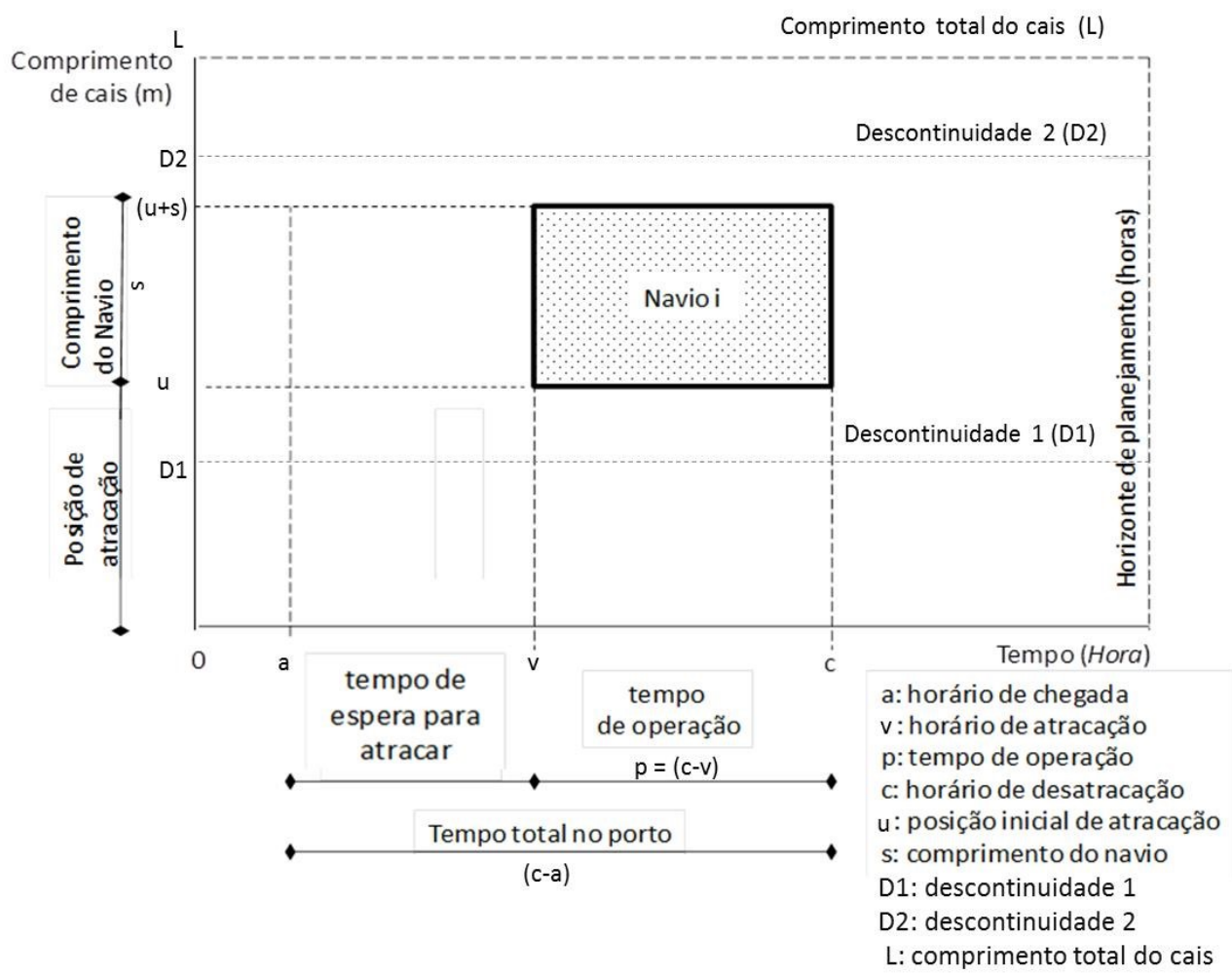

Figura 5. Parâmetros e variáveis do modelo proposto

A Figura 5 mostra a estrutura espacial e temporal dos parâmetros e variáveis utilizados no modelo proposto. No gráfico espaço-tempo, o retângulo, que simboliza o navio, não pode sobrepor às linhas de descontinuidade e ultrapassar o comprimento limite do cais.

O modelo matemático proposto é apresentado em cinco partes: conjunto, parâmetros, variáveis, função objetivo e restrições.

Conjunto

$N$ : Conjunto de navios;

Parâmetros

$L$ : Comprimento total do cais (Cais Total);

$T$ : Horizonte de tempo ou Horizonte de planejamento;

$p_{i}$ : Tempo de operação do navio $i \in N$ em unidade de tempo (1 hora);

$s_{i}$ : Comprimento do navio $i \in N$ em unidade de cais (1 metro) já incluindo a folga entre navios;

$a_{i}$ : Tempo de chegada do navio $i \in N$ ao porto;

$D_{1}$ : Ponto de descontinuidade 1 ;

$D_{2}$ : Ponto de descontinuidade 2 .

Variáveis

$u_{i}$ : Tempo de inicio/atracação do navio $i \in N$;

$v_{i}$ : Posição inicial de atracação do navio $i \in N$;

$c_{i}$ :Tempo de término/desatracação do navio $i \in N$;

$X_{i j}$ : seu valor é igual a 1 se o navio $j \in N$ atracar totalmente à direita do período ocupado pelo navio $i \in N$ no diagrama espaço-tempo. Caso contrário, o valor é 0

$Y_{i j}$ : seu valor é igual a 1 se o navio $j \in N$ atracar totalmente acima do espaço ocupado pelo navio $i \in N$ no diagrama espaço-tempo. Caso contrário, o valor é 0 . $\sigma_{i}$ : seu valor é igual a 1 se o navio $i \in N$ está atracado no segmento 1 (Berço 103 - Cais Comercial de Vitória). Caso contrário, o valor é 0 .

$\theta_{i}$ : seu valor é igual a 1 se o navio $i \in N$ está atracado no segmento 2 (Berço 101 e 102 - Cais Comercial de Vitória). Caso contrário, o valor é 0 .

$\lambda_{i}$ : seu valor é igual a 1 , se o navio $i \in N$ está atracado no segmento 3 (Terminal CODESA). Caso contrário, o valor é 0 .

A função objetivo (1) visa minimizar o tempo total de permanência dos navios no porto, ou seja, busca minimizar a diferença entre o horário de desatracação e o horário de chegada ao porto de cada navio, maximizando assim a taxa de ocupação do cais.

As restrições (2) garantem que não haja sobreposição no tempo, e as restrições (3) asseguram que não ocorra sobreposição no espaço. As restrições (4) garantem a atracação dos navios ao impor que ao menos uma das variáveis binárias $X_{i j}$ e $Y_{i j}$ seja igual a 1 .

As restrições (5) garantem o cálculo correto do tempo de desatracação do navio que é função do início da operação e do tempo de carregamento. As restrições (6) asseguram que o navio será atracado dentro do horizonte de tempo de planejamento, isto é, depois do seu instante de chegada e antes do horizonte de planejamento.

As restrições (7) eliminam a possibilidade de o navio atracar em mais de uma parte do Cais Total simultaneamente: ou ele atraca no segmento 1 (Berços 101 e 102), ou no segmento 2 (Berço 103), ou no segmento 3 (Terminal CODESA).

As restrições (8) definem que o limite inferior de posição do navio i depende da posição na qual ele está atracado. Se $\theta_{i}$ for igual a 1 , o navio está atracado no cais $\theta$ (Berços 101 e 102) e seu limite inferior de posição é 0. Se $\sigma_{i}$ for igual a 1 , o navio está atracado no cais $\sigma$ (Berço 
Função Objetivo

$\operatorname{Min} \sum_{i \in N}\left(c_{i}-a_{i}\right)$

Quadro 1. Função objetivo e restrições

Sujeito a:

$$
\begin{array}{ll}
u_{j}-u_{i}-p_{i}-\left(X_{i j}-1\right) T \geq 0 & \forall i, j \in N, j \neq i \\
v_{j}-v_{i}-s_{i}-\left(Y_{i j}-1\right) L \geq 0 & \forall i, j \in N, j \neq i \\
X_{i j}+X_{j i}+Y_{i j}+Y_{j i} \geq 1 & \forall i, j \in N, j \neq i \\
u_{i}+p_{i}=c_{i} & \forall i \in N \\
a_{i} \leq u_{i} \leq\left(T-p_{i}\right) & \forall i \in N \\
\sigma_{i}+\theta_{i}+\lambda_{i}=1 & \forall i \in N \\
v_{i} \geq\left(1-\theta_{i}\right)\left(\frac{D_{1}+D_{2}}{2}\right)+\left(1-\sigma_{i}\right)\left(\frac{D_{2}-D_{1}}{2}\right)+\left(1-\lambda_{i}\right)\left(\frac{D_{1}-D_{2}}{2}\right) & \forall i \in N \\
v_{i} \leq\left(1-\theta_{i}\right)\left(\frac{L+D_{2}-D_{1}-s_{i}}{2}\right)+\left(1-\sigma_{i}\right)\left(\frac{L+D_{1}-D_{2}-s_{i}}{2}\right)+\left(1-\lambda_{i}\right)\left(\frac{D_{1}+D_{2}-L-s_{i}}{2}\right) & \forall i \in N \\
X_{i j}, Y_{i j} \in\{0,1\} & \forall i, j \in N, j \neq i \\
\sigma_{i}, \theta_{i}, \lambda_{i} \in\{0,1\} & \forall i \in N \\
u_{i}, v_{i}, c_{i} \in \mathfrak{R}^{+} & \forall i \in N
\end{array}
$$

103) e seu limite inferior de posição é $D_{1}$. Se $\lambda_{i}$ for igual a 1 , significa que o navio está atracado no cais $\lambda$ (Terminal CODESA) e seu limite inferior de posição é $D_{2}$.

As restrições (9) também impõem limites de posição de acordo com o local de atracação dos navios. O limite imposto, neste caso, é o superior. Para o cais $\theta$ o limite superior é $\left(D_{1}-s_{i}\right)$, para o cais $\sigma$ o limite superior é $\left(D_{2}-s_{i}\right)$ e para o cais $\lambda$, esse limite superior é $\left(L-s_{i}\right)$.

As restrições (10), (11) e (12) definem o domínio das variáveis.

\section{APRESENTAÇÃO E ANÁLISE DE RESULTADOS}

Como fonte primária de dados foram utilizadas as informações do "Demonstrativo Operacional do Porto de Vi- tória de 2013”, fornecido pela Coordenação de Planejamento e Desenvolvimento - COPLAD do Porto de Vitória. Os dados que foram levantados junto à CODESA correspondem a todas as atracações, 275 navios, do ano de 2013 no Porto de Vitória (Cais Comercial de Vitória e Terminal CODESA).

Foram elaborados doze cenários diferentes para avaliar o modelo proposto, sendo que cada cenário corresponde a todas as atracações que ocorreram em cada mês do ano de 2013. As informações de todos os cenários estão disponibilizadas na Tabela 1. O Horizonte de planejamento foi calculado considerando 30 dias mais 3 para poder acomodar um eventual navio que chegou dentro do mês, porém sua desatracação ocorreu no mês subsequente.

Foi utilizado o solver CPLEX 12.6 para a execução do modelo matemático. Foi estabelecido como tempo limite 14.400 segundos (4 horas) para resolução de cada um dos modelos associados aos cenários, usando um computador Intel i7, com 8GB de memória RAM.

Tabela 1. Cenários Estudados CAA

\begin{tabular}{cccc}
\hline Cenário & Mês & $\begin{array}{c}\text { Quantidade } \\
\text { de navios } \\
\text { (un) }\end{array}$ & $\begin{array}{c}\text { Horizonte de } \\
\text { Planejamento } \\
\text { (horas) }\end{array}$ \\
\hline 1 & Janeiro & 17 & 792 \\
2 & Fevereiro & 19 & 792 \\
3 & Março & 29 & 792 \\
4 & Abril & 14 & 792 \\
5 & Maio & 25 & 792 \\
6 & Junho & 31 & 792 \\
7 & Julho & 27 & 792 \\
8 & Agosto & 18 & 792 \\
9 & Setembro & 20 & 792 \\
10 & Outubro & 23 & 792 \\
11 & Novembro & 27 & 792 \\
12 & Dezembro & 26 & 792 \\
\hline
\end{tabular}


Planejamento de Atracação de Navios por Meio de um Modelo Matemático para o

Problema de Alocação de Berços Contínuos com Descontinuidade do Cais

Tabela 2. Resultados apresentados pelo CPLEX para os cenários

\begin{tabular}{|c|c|c|c|c|c|c|c|c|}
\hline & \multicolumn{4}{|c|}{ Modelo PABC-DC (CPLEX) } & \multicolumn{2}{|c|}{ Caso Real } & \multicolumn{2}{|c|}{ Redução } \\
\hline Cenário & GAP (\%) & TEX (s) & TMA (h) & TME (h) & TMA (h) & TME (h) & TMA (\%) & TME (\%) \\
\hline$(1)$ & $(2)$ & (3) & (4) & (5) & (6) & (7) & (8) & (9) \\
\hline 1 & 0,00 & $3.786,90$ & 6,45 & 32,88 & 7,53 & 34,24 & 14,34 & 3,97 \\
\hline 2 & 0,00 & $3.897,56$ & 7,89 & 50,16 & 8,71 & 53,58 & 9,41 & 6,39 \\
\hline 3 & 4,35 & $14.400,00$ & 10,90 & 46,66 & 11,20 & 49,24 & 2,68 & 5,25 \\
\hline 4 & 0,00 & $2.546,89$ & 4,78 & 23,21 & 4,89 & 25,43 & 2,25 & 8,71 \\
\hline 5 & 0,00 & $12.789,98$ & 9,89 & 39,20 & 10,90 & 42,36 & 9,27 & 7,46 \\
\hline 6 & 5,78 & $14.400,00$ & 13,56 & 42,71 & 15,49 & 46,57 & 12,46 & 8,29 \\
\hline 7 & 2,89 & $14.400,00$ & 10,34 & 44,57 & 11,27 & 47,58 & 8,25 & 6,33 \\
\hline 8 & 0,00 & $4.567,90$ & 8,67 & 29,83 & 9,34 & 32,33 & 7,17 & 7,72 \\
\hline 9 & 0,00 & $5.254,89$ & 5,78 & 32,11 & 6,57 & 34,57 & 12,02 & 7,13 \\
\hline 10 & 0,00 & $9.578,90$ & 11,45 & 36,96 & 11,90 & 38,92 & 3,78 & 5,04 \\
\hline 11 & 3,79 & $14.400,00$ & 12,10 & 32,37 & 13,97 & 33,35 & 13,39 & 2,94 \\
\hline 12 & 2,34 & $14.400,00$ & 7,67 & 30,35 & 9,45 & 31,24 & 18,84 & 2,86 \\
\hline
\end{tabular}

Os resultados obtidos com o modelo matemático proposto foram comparados com aqueles empregados pelos terminais estudados (processo empírico, manual), que neste artigo são definidos como resultados do Caso Real.

Na Tabela 2 são apresentados os resultados alcançados pelo CPLEX, os resultados associados às soluções empregadas pelos terminais (Caso Real) e a redução alcançada quando os dois resultados são comparados. A coluna 1 representa os cenários analisados. As colunas 2, 3, 4 e 5 apresentam os valores alcançados pelo CPLEX com o modelo matemático proposto PABC-DC, respectivamente, o GAP residual obtido após 4 horas de processamento, o Tempo de Execução do CPLEX (TEX), o Tempo Médio de Espera para Atracar (TMA) e o Tempo Médio Total de Estadia do Navio no Porto (TME). Para o planejamento realizado pelo porto, Caso Real, nas colunas 6 e 7 são apresentados o Tempo Médio de Espera para Atracar (TMA) e o Tempo Médio Total de Estadia do Navio no Porto (TMP), respectivamente.

Nas colunas 8 e 9 são apresentadas as reduções percentuais alcançadas pelos resultados gerados pelo CPLEX em relação aos gerados pelo planejamento realizado pelo porto. Têm-se, então, nas colunas 8 e 9, respectivamente, a redução do Tempo Médio de Espera para Atracar (TMA) e a redução do Tempo Médio Total de Estadia do Navio no Porto (TMP). O Tempo Médio Total de Estadia no Porto engloba o Tempo Médio de Espera para Atracação mais o tempo de operação do navio.

Pela Tabela 2, pode-se verificar que o CPLEX com o modelo matemático proposto alcançou resultados melhores para todos os 12 cenários nas duas métricas adotadas: Tempo Médio de Espera para Atracar e Tempo Médio de Estadia no porto. Mesmo nos Cenários 3, 6, 7, 11 e 12 que apresentaram GAP residual, ou seja, o CPLEX parou sem garantir a solução ótima devido ao tempo limite para processamento, verifica-se que as melhores soluções encontradas alcançaram valores melhores quando comparados com os resultados gerados pelo processo atualmente empregado nos terminais.

Pode-se notar que no Cenário 6, o CPLEX alcançou uma redução de 8,3\% do tempo total de estadia no porto, mesmo tendo apresentado um GAP de 5,78\%. A redução alcançada está relacionada à redução do tempo de espera para atracação, coluna 8 da Tabela 2, e também, quando analisados em detalhes os resultados, à uma melhor distribuição dos navios ao longo da extensão do Cais Total. As outras reduções alcançadas nos outros cenários estão associadas aos mesmos motivos apresentados para o Cenário 6.

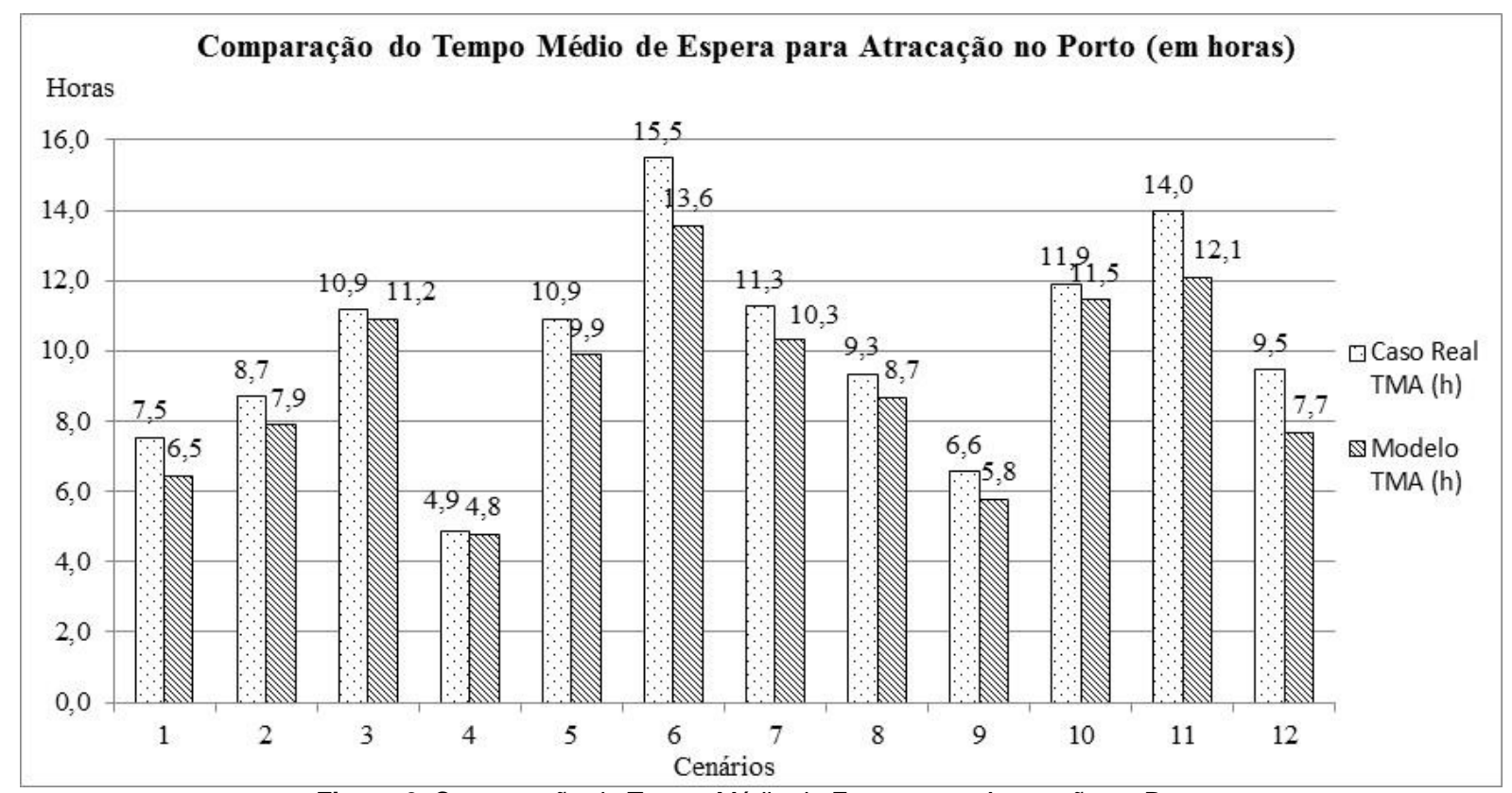

Figura 6. Comparação do Tempo Médio de Espera para Atracação no Porto 


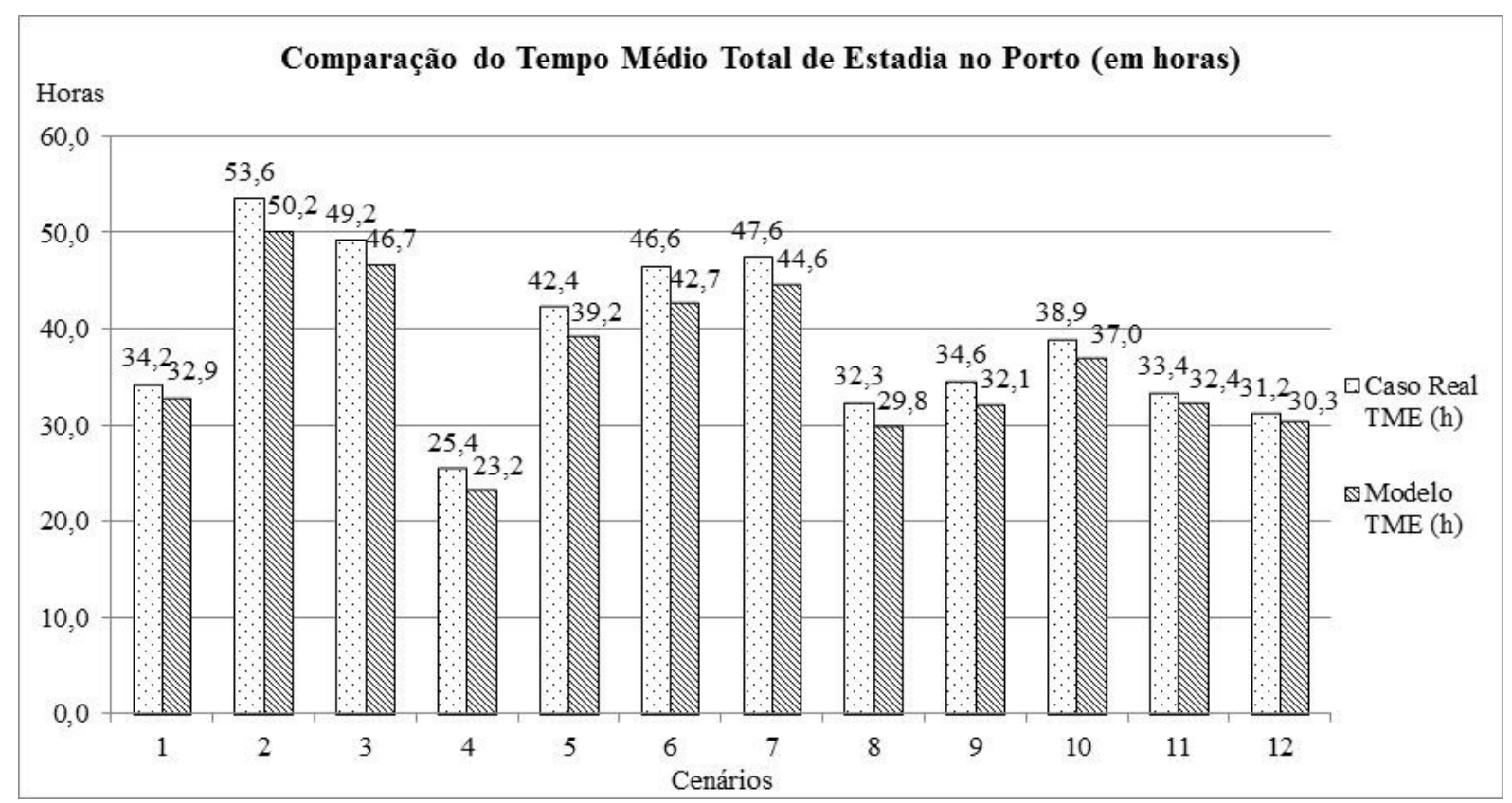

Figura 7. Comparação do Tempo Médio Total de Estadia no Porto

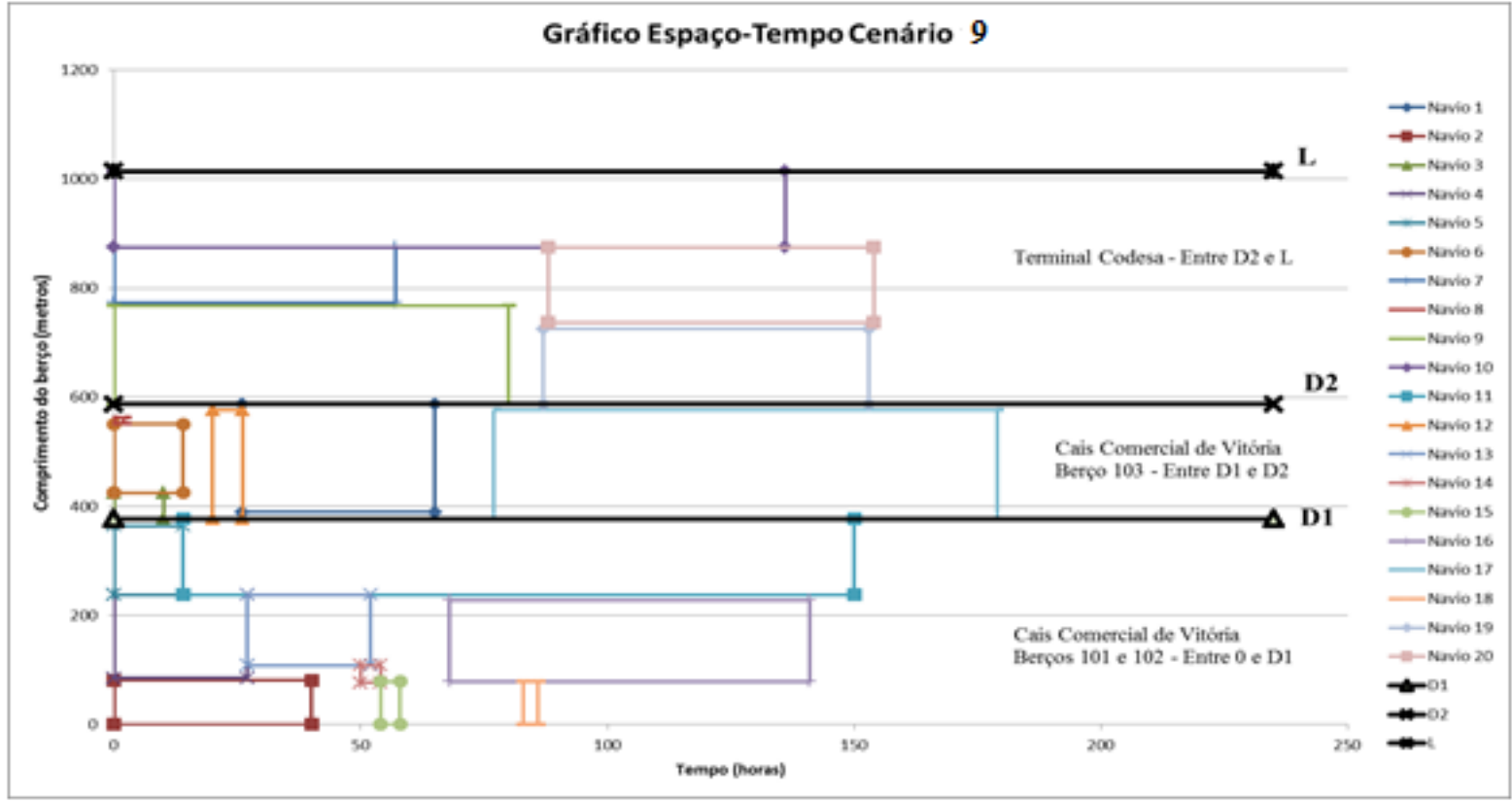

Figura 8. Programação de Atracações para o Cenário 9

Apesar dos valores de redução apresentados para espera média para atracar, coluna 8 , serem altos em termos percentuais, quando se analisa em termos de horas, os valores continuam importantes, embora menores. Subtraindo os valores das colunas 6 e 4 da mesma tabela, pode-se verificar que os ganhos em horas variam de 0,3 a 1,9.

Pode-se verificar no gráfico da Figura 6 que o modelo proposto conseguiu resultados melhores para o tempo de espera para atracação em todos os cenários.

No gráfico da Figura 7 é realizada a comparação dos resultados referentes ao tempo médio total de estadia no porto alcançados pelo modelo e pelo processo empregado pelos terminais. Pode-se notar que houve redução no tempo total de estadia dos navios no porto em todos cenários analisados.

Somando-se todas as diferenças entre o resultado do modelo e do processo manual para cada um dos 275 navios que atracaram no Porto de Vitória, obteve-se uma redução total de 663,11 horas/ano. Esta redução equivale a um ga- nho de 27,63 dias/ano, o que equivale dizer que o porto ganharia aproximadamente o equivalente a um mês durante o ano, podendo atrair outros navios para o porto e com isso aumentar sua receita.

Tomando como base que um navio típico que atraca no Porto de Vitória tem um custo que gira na ordem de US\$30.000,00/dia, pode-se inferir que caso a CODESA tivesse usado o modelo matemático proposto para planejar a atracação dos navios no ano de 2013 ela teria conseguido uma redução de custos para os armadores dos navios na ordem de US\$19.893.142,11. Isso tornaria o porto mais atrativo para os armadores podendo fomentar a vinda de novas rotas comerciais para o Estado e também mais receitas para o porto.

Usualmente, na prática dos portos, o planejamento da programação de navios fica restrito a um Horizonte de planejamento de uma semana, ou seja, o modelo matemático proposto alcançou um resultado ótimo, ou próximo do ótimo, para uma programação com Horizonte de planejamento quatro vezes maior que o usual, 33 dias, e mesmo nos 
casos em que a solução ótima não foi obtida, o CPLEX alcançou resultados sempre melhores que o processo empregado pelos terminais.

A Figura 8 apresenta o gráfico espaço-tempo para o Cenário 9, mês de setembro. Pode-se perceber que solução encontrada para os 20 navios não apresenta qualquer tipo de sobreposição no espaço ou no tempo, como esperado. As descontinuidades foram respeitadas, ou seja, os navios não ultrapassaram os limites D1, D2, e L. Assim, o modelo conseguiu planejar atracações de forma integrada para os três segmentos de atracação dos terminais estudados de modo a minimizar o tempo total gasto pelos navios no porto.

\section{CONCLUSÃO}

Este artigo tratou um problema real de um porto, com margens separadas e descontinuidade em um dos cais. Mesmo com essas divisões, a programação de atracação dos navios deve ser realizada de modo a integrar no planejamento todas essas descontinuidades e proporcionar o planejamento integrado de toda a extensão de cais do porto.

Para isso, foi proposto um modelo matemático que difere dos demais vistos na literatura, por tratar dos quesitos citados e ser resolvido de maneira integrada. O modelo, baseado em Programação Linear Inteira Mista, busca minimizar o tempo total do navio no porto, considerando-se o cais contínuo com descontinuidades e com chegada dinâmica que foi denominado como Problema de Alocação de Berços com Descontinuidade de Cais (PABC-DC).

$\mathrm{O}$ modelo proposto foi aplicado aos Terminais do Porto de Vitória: Cais Comercial de Vitória e Terminal CODESA, ambos gerenciados pela CODESA, e mostrou bons resultados. Foi possível identificar que os resultados são condizentes com o que hoje é planejado e, sobretudo, apresentam ganhos para a CODESA, que podem representar uma maior disponibilidade de cais e um nível de serviço maior para os navios com a redução do seu tempo de permanência no porto.

É importante destacar que o modelo pode ser aplicado a diversos portos do Brasil e do mundo que possuam duas margens ou qualquer detalhe de projeto que impeça a atracação de forma contínua.

Pelos resultados alcançados, sabe-se que o modelo proposto resolvido de forma exata com o CPLEX, consegue resolver instâncias de pequena e média escala, que representam a realidade do porto estudado. Para resolver instâncias de maior escala, que correspondem a portos de maior movimentação de navios, está sendo desenvolvida uma meta-heurística para o modelo proposto.

\section{AGRADECIMENTOS}

Os autores agradecem à FAPES (458/2013), ao CNPq (477357/2013-0) e à CAPES pelo apoio financeiro e a CODESA pelo apoio ao fornecimento dos dados do problema.

\section{REFERÊNCIAS}

ANTAQ (2013) Boletim Portuário $1^{\circ}$ Semestre 2013. Agência Nacional de Transportes Aquaviários.
Bierwirth, C.; Meisel, F. (2009) A fast heuristic for quay crane scheduling with interference constraints. Journal of Scheduling, v. 12, n. 4, p. 345-360. DOI: 10.1007/s10951-009-0105-0

Bierwirth, C.; F. Meisel (2010) A survey of berth allocation and quay crane scheduling problems in container terminals. European Journal of Operational Research, v. 202, n. 3, p. 615- 627. DOI: 10.1016/j.ejor.2009.05.031

Briano, C.; Briano, E.; Bruzzone, A.G. (2005) Models for support maritime logistics: a case study for improving terminal planning. In: Merkuryev, Y., Zobel, R., Kerckhoffs, E. (eds.) Proceedings of the 19th European Conference on Modeling and Simulation (ECMS). p. 199-203.

Brown, G.G.; Cormican, K.J.; Lawphongpanich, S.; Widdis, D. (1997) Optimizing submarine berthing with a persistence incentive. Naval Research Logistics, v. 44, n. 4, p. 301-318. DOI: 10.1002/(SICI)1520-6750(199706)44:4<301::AID-AV2>3.0.CO; 2-A

Brown, G.G.; Lawphongpanich, S.; Thurman, K.P. (1994) Optimizing ship berthing. Naval Research Logistics, v. 41, n. 1, p. 115. DOI: 10.1002/1520-6750(199402)41:1<1::AID NAV3220410102>3.0.CO;2-L

Chang, D.; Yan, W.; Chen, C.-H.; Jiang, Z. (2008) A berth allocation strategy using heuristics algorithm and simulation optimization. International Journal of Computer Applications in Technology, v. 32, n. 4, p. 272-281. DOI: 10.1504/IJCAT.2008.021382

Chen, C.-Y.; Hsieh, T.-W. (1999) A time-space network model for the berth allocation problem. In: 19th IFIP TC7 Conference on System Modeling and Optimization, Cambridge, England, p. 12 16.

Cheong, C.; Tan, K.; Liu, D.; Lin, C. (2010) Multi-objective and prioritized berth allocation in container ports. Annals of Operations Research, v. 180, n. 1, p. 63-103. DOI: 10.1007/s10479-008 0493-0

Cheong, C.Y.; Lin, C.J.; Tan, K.C.; Liu, D.K. (2007) A multi-objective evolutionary algorithm for berth allocation in a container port. In: Congress on Evolutionary Computation 2007 (CEC 2007). IEEE, Singapore, p. 927-934. DOI: 10.1109/CEC.2007.4424569

CODESA. (2014a) Complexo Portuário. Companhia Docas do Espírito Santo. Disponível em: http://www.CO DESA.gov.br/site/OPorto/ComplexoPortu\%C3\%A1rio/tabid/77/1 anguage/pt-BR/Default.aspx. Acesso em 05/03/2014.

CODESA. (2014b) Anuário Estatístico de 2013. Companhia Docas do Espírito Santo. Disponível em: http://www.vitoria port.com.br/Site/LinkClick.aspx?fileticket=piEIiUcsB8\%3D\&tabid=665\&mid=1589\&language=pt-BR. Acesso em 10/05/2014.

Cordeau, J.-F.; Laporte, G.; Legato, P.; Moccia, L. (2005) Models and tabu search heuristics for the berth-allocation problem. Transportation Science, v. 39, n. 4, p. 526-538. DOI: 10.1287/trsc. 1050.0120

Dai, J.; Lin, W.; Moorthy, R.; Teo, C.-P. (2008) Berth allocation planning optimization in container terminals. In: Tang, C.S.; Teo, C.-P.; Wei, K.-K. (eds.) Supply chain analysis: a handbook on the interaction of information, System and Optimization. Springer, New York, p. 69-105. DOI: 10.1007/978-0-387-75240-2_4

Elwany, M. H.; Ali, I.; Abouelseoud, Y. (2013) A heuristics-based solution to the continuous berth allocation and crane assignment 
problem. Alexandria Engineering Journal, v. 52, n. 4, p. 671-677. DOI: 10.1016/j.aej.2013.09.001

Ganji, S. R. S.; Babazadeh, A; Arabshahi, N. (2010) Analysis of the continuous berth allocation problem in container ports using a genetic algorithm. Journal of marine science and technology, $\mathrm{v}$. 15, n. 4, p. 408-416. DOI: 10.1007/s00773-010-0095-9

Garey, M.R.; Johnson, D.S. (1979) Computers and intractability: a guide to the theory of np-completeness. WH Freeman, San Francisco, USA.

Goh, K.S.; Lim, A. (2000) Combining various algorithms to solve the ship berthing problem. In: Proceedings of the 12th International Conference on Tools with Artificial Intelligence (ICTAI'00). IEEE, Vancouver, BC, p. 370-375. DOI: 10.1109/TAI.2000.889896

Guan, Y.; Cheung, R.K. (2004) The berth allocation problem: models and solution methods. OR Spectrum, v. 26, n. 1, p.75-92. DOI: $10.1007 / \mathrm{s} 00291-003-0140-8$

Guan, Y.; Xiao, W.-Q.; Cheung, R.K.; Li, C.-L. (2002) A multiprocessor task scheduling model for berth allocation: heuristic and worst-case analysis. Operations Research Letters, v. 30, n. 5, p. 343-350. DOI: 10.1016/S0167-6377(02)00147-5

Imai, A.; Nishimura, E.; Hattori, M.; Papadimitriou, S. (2007) Berth allocation at indented berths for mega-containerships. European Journal of Operational Research, v. 179, n. 2, p. 579-593. DOI: 10.1016/j.ejor.2006.03.034

Imai, A.; Nishimura, E.; Papadimitriou, S. (2001) The dynamic berth allocation problem for a container port. Transportation Research Part B: Methodological, v. 35, n. 4, p. 401-417. DOI: 10.1016/S0191-2615(99)00057-0

Imai, A.; Sun, X.; Nishimura, E.; Papadimitriou, S. (2005) Berth allocation in a container port: using a continuous location space approach. Transportation Research Part B: Methodological, v. 39, n. 3, p. 199-221. DOI: 10.1016/j.trb.2004.04.004

Kim, K.H.; Moon, K.C. (2003) Berth scheduling by simulated annealing. Transportation Research Part B: Methodological, v. 37, n. 6, p. 541-560. DOI: 10.1016/S0191-2615(02)00027-9

Kim, K. H.; Park, Y. M. (2004) A crane scheduling method for port container terminals. European Journal of Operational Research, v. 156, n. 3, p. 752-768. DOI: 10.1016/S03772217(03)00133-4

Lee, Y.; Chen, C.-Y. (2008) An optimization heuristic for the berth scheduling problem. European Journal of Operational Research, v. 196, n. 2, p. 500-508. DOI: 10.1016/j.ejor.2008.03.021

Li, C.-L.; Cai, X.; Lee, C.-Y. (1998) Scheduling with multiplejob-on-one-processor pattern. IIE Transactions, v. 30, n. 5, p. 433445. DOI: 10.1023/A:1026487022192

Lim, A. (1998) The berth planning problem. Operations Research Letters, v. 22, n. 2, p. 105-110. DOI: 10.1016/S0167 6377(98)00010-8

Lim, A. (1999) An effective ship berthing algorithm. In: Thomas, D. (ed.) Proceedings of the 16th International Joint Conference on Artificial Intelligence (IJCAI-99-vol-1). Morgan Kaufmann Publishers, San Francisco, p. 594-599.

Meersmans, P.J.M.; Dekker, R. (2001) Operations research supports container handling. Econometric Institute Research Papers, v. 234.
Moon, K. (2000) A mathematical model and a heuristic algorithm for berth planning. Brain Korea, v. 21.

Moorthy, R.; Teo, C.-P. (2006) Berth management in container terminal: the template design problem. OR Spectrum, v. 28, n. 4, p. 495-518. DOI: 10.1007/978-3-540-49550-5_4

Nishimura, E.; Imai, A.; Papadimitriou, S. (2001) Berth allocation planning in the public berth system by genetic algorithms. European Journal of Operational Research, v. 131, n. 2, p. 282-292. DOI: 10.1016/S0377-2217(00)00128-4

Park, K.T.; Kim, K.H. (2002) Berth scheduling for container terminals by using a sub-gradient optimization technique. Journal of the Operational Research Society, v. 53, n. 9, p. 1054-1062. DOI: 10.1057/palgrave.jors.2601412

Park, Y.M.; Kim, K.H. (2003) A scheduling method for berth and quay cranes. OR Spectrum, v. 25, n. 1, p. 1-23. DOI: 10.1007/s00291-002-0109-z

Rashidi, H.; Tsang, E. (2013) Novel constraints satisfaction models for optimization problems in container terminals. Applied Mathematical Modelling, v. 37, n. 6, p. 3601-3634. DOI: 10.1016/j.apm.2012.07.042

Stahlbock, R.; Voß, S. (2008) Operations research at container terminals: a literature update. OR Spectrum, v. 30, n. 1, p. 1-52. DOI: 10.1007/s00291-007-0100-9

Steenken, D.; Voß, S.; Stahlbock, R. (2004) Container terminal operation and operations research - a classification and literature review. OR Spectrum, v. 26, n. 1, p. 3-49. DOI: 10.1007/s00291 003-0157-z

Tang, L.; Lia, S.; Liub, J. (2009) Dynamically scheduling ships to multiple continuous berth spaces in an iron and steel complex. International Transactions in Operational Research, v. 16, n. 1, p. 87-107. DOI: 10.1111/j.1475-3995.2009.00662.x

Tong, C.J.; Lau, H.C.; Lim, A. (1999) Ant colony optimization for the ship berthing problems. In: Thiagarajan, P.S.; Yap, R. (eds.) Advances in Computing Science - ASIAN'99: Fifth Asian Computing Science Conference, v. 1742 of LNCS. Springer Berlin Heidelberg, p. 359-370. DOI: 10.1007/3-540-46674-6_30

Umang, N.; Bierlaire, M. (2012) Real Time Recovery in Berth Allocation Problem in Bulk Ports. In: Proceedings of the Swiss Transport Research Conference (STRC).

Umang, N.; Bierlaire, M.; Vacca, I. (2013) Exact and heuristic methods to solve the berth allocation problem in bulk ports. Transportation Research Part E: Logistics and Transportation Review, v. 54, p. 14-31. DOI: 10.1016/j.tre.2013.03.003

Vacca, I.; Bierlaire, M.; Salani, M. (2007) Optimization at container terminals: Status, trends and perspectives. In: Proceedings of the Swiss Transport Research Conference (STRC), p. 1-21.

Vis, I.F.A. ; Koster, R. de (2003) Transshipment of containers at a container terminal: an overview. European Journal of Operational Research, v. 147, n. 1, p. 1-16. DOI: 10.1016/S0377 2217(02)00293-X

Wang, F.; Lim, A. (2007) A stochastic beam search for the berth allocation problem. Decision Support Systems, v. 42, n. 4, p. 2186 2196. DOI: 10.1016/j.dss.2006.06.008. 